\title{
Editorial
}

\section{Modeling, Optimization, and Verification for Complex Systems}

\author{
Guiming Luo, ${ }^{1}$ Xiaoyu Song, ${ }^{2}$ Xiaojing Yang, ${ }^{3}$ Krishnaiyan Thulasiraman, ${ }^{4}$ \\ Jean-François Monin, ${ }^{5}$ and Guowu Yang ${ }^{6}$ \\ ${ }^{1}$ School of Software, Tsinghua University, Beijing 100084, China \\ ${ }^{2}$ Department of Electrical and Computer Engineering, Portland State University, P.O. Box 751, Portland, OR 97207, USA \\ ${ }^{3}$ Department of Mathematical Sciences, Tsinghua University, Beijing 100084, China \\ ${ }^{4}$ School of Computer Science, University of Oklahoma, 200 Felgar Street, Room 114, Norman, OK 73019, USA \\ ${ }^{5}$ Verimag, Université Grenoble Alpes, France \\ ${ }^{6}$ School of Computer Science and Engineering, University of Electronic Science and Technology of China, Chengdu, \\ Sichuan 611731, China \\ Correspondence should be addressed to Guiming Luo; gluo@tsinghua.edu.cn
}

Received 28 December 2015; Accepted 28 December 2015

Copyright (C) 2016 Guiming Luo et al. This is an open access article distributed under the Creative Commons Attribution License, which permits unrestricted use, distribution, and reproduction in any medium, provided the original work is properly cited.

System modeling tends to have many complex features, uncertainty influencing many important aspects and complications. It is a challenge to construct an unchanging mathematical model. Intelligent modeling embeds novel frontier sciences and technologies, such as data modeling, abstract modeling, automatic modeling and verification, and computer simulation.

Significant achievements have been made in system modeling and verification in recent years. This special issue concentrates on current developments in the areas of systems modeling, intelligent computing, and formal verification. It has been warmly supported by the authors and more than 120 manuscripts were received. After the high-quality peerreviewed process, 33 papers have been selected and published.

The internal structure of Bivium was exploited and generalized to an algorithm, Bivium-Model, by S. Zhang et al. in the paper "Enhanced-Bivium Algorithm for RFID System." Model-driven engineering has played an essential role in software development. The paper by Z. Zhang et al. entitled "Composite-Level Conflict Detection in UML Model Versioning" presents an approach to conflict detection at the composite level in model versioning systems for Unified Modeling Language. A new approach of choreography model verification based on Description Logic is presented in the paper "Verifying Service Choreography Model Based on Description Logic" by M. Yu et al. A metamodel of service choreography is built to provide a conceptual framework to capture the formal syntax and semantics of service choreography. Machine theorem proving is one of the formal methods that perform accurate analysis with completeness to some extent. In the paper by Z. Shi et al., "The Formalization of Discrete Fourier Transform in HOL", the authors propose the formalization of DFT in a higher-order-logic theorem prover named HOL. Given the continuous nature of kinematic analysis and the shortcoming of the traditional verification methods, A. Wu et al. in the paper entitled "Formal Kinematic Analysis of a General 6R Manipulator Using the Screw Theory" utilize the geometrical theory of HOL4 to develop formal reasoning support for the kinematic analysis of a robotic manipulator and conduct the formal kinematic analysis of a general $6 \mathrm{R}$ manipulator by high-order-logic theorem proving. In the work by J. Fu et al. "A Deductive Approach towards Reasoning about Algebraic Transition Systems," the authors present a deductive approach for specifying and verifying algebraic transition systems. The semantics of modalities and formulas is defined with solutions of algebraic equations. A proof system is constructed to verify properties of algebraic transition systems.

How to find the optimal transportation route in sea-trade is very important for the logistics industry. Facing the huge network extracted from the foreign trading industry as well as the complex constraints, it is impossible for the traditional 
optimization methods to find the solution in a short time, which is motivated in the paper entitled "A Graph Based Framework for Route Optimization in Sea-Trade Logistics" by $\mathrm{D}$. Chu et al. The incremental optimization of the capacitated single-allocation hub and spoke network problem is considered in the paper entitled "Incremental Optimization of Hub and Spoke Network for the Spokes' Numbers and Flow" by Y. Wang and Y. Huang. Two mixed-integer linear programming formulations are proposed and analyzed. The paper by S. Gaona and D. Romero titled "TPA: A Two-Phase Approach Using Simulated Annealing for the Optimization of Census Taker Routes in Mexico" presents a Two-Phase Approach (TPA) to optimize the routes of INEGI's census takers. A tuning methodology based on the irace package is used to determine the parameter values for TPA on a subset of 150 instances provided by INEGI. With the UAV aerial images from a mountainous area in China, $\mathrm{C}$. $\mathrm{Xu}$ et al. in the paper entitled "3D Reconstruction of Tree-Crown Based on the UAV Aerial Images" present an algorithm for $3 \mathrm{D}$ reconstruction of tree-crown. A feature area extraction method is proposed based on watershed segmentation, and the local area correlation coefficient is calculated to match the feature area.

In "Numerical Simulation and Experimental Study of Deep Bed Corn Drying Based on Water Potential" by Z. Liu et al., the authors establish a numerical simulation system of deep bed corn drying process which has been proved to be effective according to the results of numerical simulation and corresponding experimental investigation and has revealed that desorption and adsorption coexist in deep bed drying. To solve the problem of parameter selection during the design of magnetically coupled resonant wireless power transmission system (MCR-WPT), the paper by S. Lu et al., "The Parameters Optimization of MCR-WPT System Based on the Improved Genetic Simulated Annealing Algorithm," proposes an improved genetic simulated annealing algorithm. The paper by C. Ma, "Estimating Frequency by Interpolation Using Least Squares Support Vector Regression," employs a modified version of support vector regression (SVR), least squares SVR, to interpolate on Fourier coefficients of received signals and attain high frequency estimation accuracy.

With advances in connected vehicle technology, dynamic vehicle route guidance models gradually become indispensable equipment for drivers. In the work by D. Tian et al. "A Dynamic Travel Time Estimation Model Based on Connected Vehicles", the authors propose a dynamic travel time estimation model and a road link dynamic dividing algorithm. Brake squeal is often analytically studied by a complex eigenvalue analysis of linearized models of the brake assembly that is usually quite large. Based on the complex modes theory, L. Zhang et al. in the paper entitled "A Reduced-Order Model for Complex Modes of Brake Squeal Model and Its Application to a Flexible Pin-on-Disc System" present a reduced-order model and a method for determining those frequencies having the most effect on the pair of coupling frequencies.

The model with fuzzy teaching preference provides a more satisfactory solution to a course assignment problem than assigning arbitrary weights. Using sensitivity analysis,
A. Gorka and P. Thipwiwatpotjana in the paper entitled "The Importance of Fuzzy Preference in Course Assignment Problem" devise a method for improving a fuzzy membership function. The paper by Y. Chen and Y. Wang, "Two-Stage Fuzzy Portfolio Selection Problem with Transaction Costs," describes a two-period portfolio selection problem. Given the first-stage decision vector and realization of fuzzy return, the optimal value expression of the second-stage programming problem is derived. Based on cellular automaton, a new static floor field method for simulations of evacuation processes was presented in the paper by X. Yang et al., "Floor Field Model Based on Cellular Automata for Simulating Indoor Pedestrian Evacuation." The authors propose concept of scenario familiarity of the crowd for explaining the model. Artificial bee colony (ABC) algorithm is a popular swarm intelligence technique inspired by the intelligent foraging behavior of honeybees. By adopting the new fitness evaluation method and combining it with the two-dimensional entropy multithreshold segmentation principle, X. Zhang et al. in the paper entitled "Flame Image Segmentation Based on the Bee Colony Algorithm with Characteristics of Levy Flights" develop a threshold segmentation test of the flame image. Combining grenade explosion method (GEM) with Cauchy operator, J.-G. Zheng et al. in the paper entitled "Artificial Bee Colony Algorithm Combined with Grenade Explosion Method and Cauchy Operator for Global Optimization" propose the ABCGC method to improve the performance of ABC Algorithm.

In the work by C. Ruan et al. entitled "Sequence and Direction Planning of Multiobjective Attack in Virtual Navigation Based on Variable Granularity Optimization Method," the Variable Granularity Hybrid Particle Swarm Optimization method is proposed and the planning problem is solved by decomposing it into attacking sequence planning and attacking direction planning. The electrical power system construction is often a pivotal part in the national and regional economic development plan. The paper by P. Jiang et al. "A New Hybrid Model Based on Data Preprocessing and an Intelligent Optimization Algorithm for Electrical Power System Forecasting" constructs a hybrid model, known as the E-MFA-BP model that can forecast indices in the electrical power system, including wind speed, electrical load, and electricity price. Based on the wind driven optimization (WDO) and differential evolution (DE), the paper by $\mathrm{Z}$. Bao et al., "A Hybrid Global Optimization Algorithm Based on Wind Driven Optimization and Differential Evolution," presents a hybrid global optimization WDO-DE algorithm. The populations of individuals both in WDO and in DE employ an information sharing mechanism to implement coevolution. Based on QoS differentiation and VM resizing, the paper by R. Hu et al., "A New Resources Provisioning Method Based on QoS Differentiation and VM Resizing in IaaS," proposes an adaptive host resource provisioning method to improve the host energy efficiency in IaaS.

The paper by J. Ye and H. Ma entitled "Multiobjective Joint Optimization of Production Scheduling and Maintenance Planning in the Flexible Job-Shop Problem" provides a scientific decision analysis method for enterprise to make production plan and maintenance plan. In the paper by $\mathrm{N}$. 
Zhao et al. "Simulation-Based Optimization for Storage Allocation Problem of Outbound Containers in Automated Container Terminals," the authors propose a simulation-based optimization method for the storage allocation problem of outbound containers in automated container terminals. The complete simulation-based optimization method is formed by the Particle Swarm Optimization and Genetic Algorithm. J. Xiong et al. in the paper entitled "Controllability of Nonlinear Impulsive Stochastic Evolution Systems Driven by Fractional Brownian Motion" consider the infinite-dimensional dynamical control systems described by nonlinear impulsive stochastic evolution differential equations. Sufficient conditions for the complete controllability of nonlinear impulsive stochastic systems are formulated and proved.

In order to give full consideration to the consumer's personal preference in cloud service selection strategies and improve the credibility of service prediction, the paper by $\mathrm{Y}$. Wang et al. entitled "CC-PSM: A Preference-Aware Selection Model for Cloud Service Based on Consumer Community" presents a preference-aware cloud service selection model based on consumer community. In the work by X. Han and S. Liu entitled "Bayes-Nash Equilibrium of the Generalized First-Price Auction," the authors analyze the generalized first-price auction under incomplete information setting. Without setting a reserve price, the efficient symmetrical Bayes-Nash equilibrium is characterized. The paper by J. Ding et al. "Modeling the Process of Event Sequence Data Generated for Working Condition Diagnosis" proposes a probabilistic generative model for simulating the process of event sequence data generated and depicting the working condition of equipment at runtime. Q. Yu et al. develop a modeling method of approximated transition system based on the approximate solution of matrix in the paper entitled "Approximate Analyzing of Labeled Transition Systems." It provides a facility to approximately formal semantic modeling for continuous-time linear systems and to effective error analyzing.

In the paper by R. Wang et al. entitled "Modeling and Analysis of the Obstacle-Avoidance Strategies for a Mobile Robot in a Dynamic Environment," the authors present the Markov Decision Process based probabilistic formal models for three obstacle-avoidance strategies of a mobile robot in an uncertain dynamic environment. S. Dai et al. in the paper entitled "A Formal Approach for RT-DVS Algorithms Evaluation Based on Statistical Model Checking" present an approach to evaluate RT-DVS algorithms using statistical model checking. A scalable framework is proposed for RT-DVS algorithms evaluation, in which the relevant components are modeled as stochastic timed automata, and the evaluation metrics including utilization bound, energy efficiency, battery awareness, and temperature awareness are expressed as statistical queries.

\section{Acknowledgments}

We sincerely thank the researchers who contributed to this special issue for their inspiring work, as well as the anonymous reviewers for their diligent assistance. In addition, we would like to express our appreciation for the editorial board members of this journal, who provided valuable help and support throughout the preparation of this special issue.

Guiming Luo

Xiaoyu Song

Xiaojing Yang

Krishnaiyan Thulasiraman

Jean-François Monin

Guowu Yang 


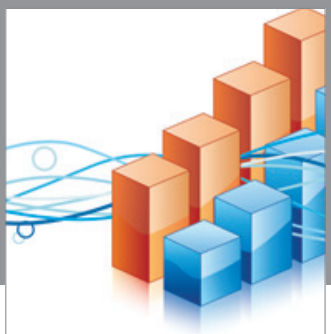

Advances in

Operations Research

vatem alat4

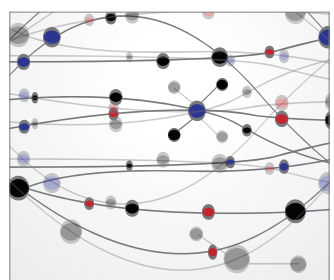

\section{The Scientific} World Journal
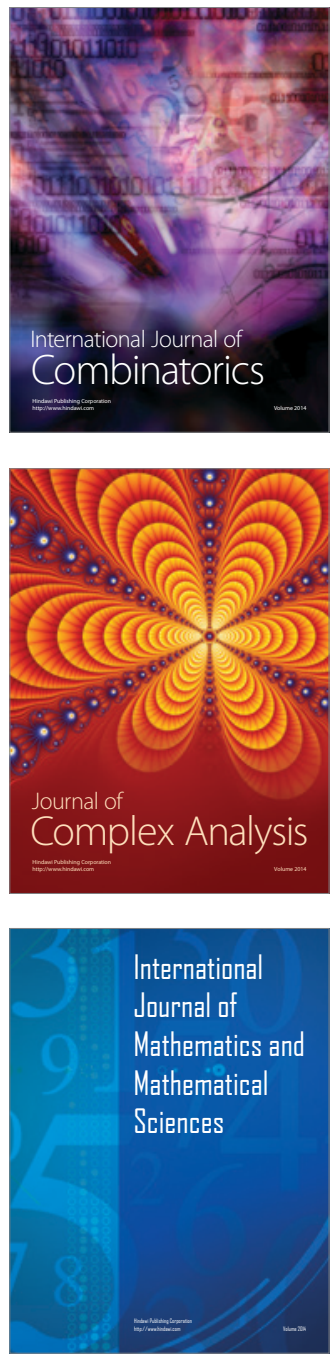
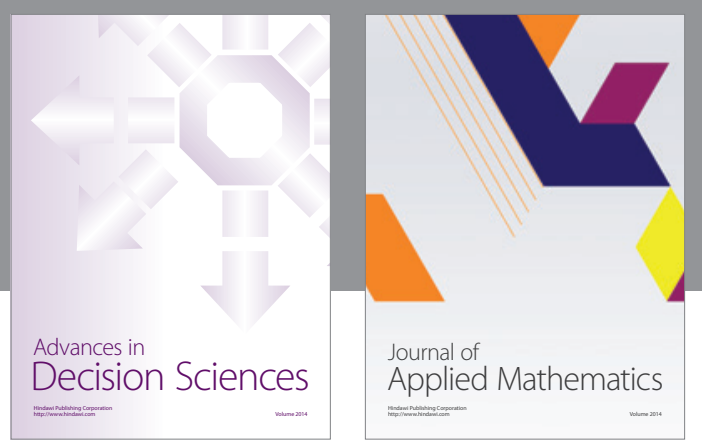

Algebra

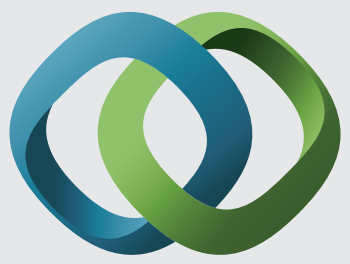

\section{Hindawi}

Submit your manuscripts at

http://www.hindawi.com
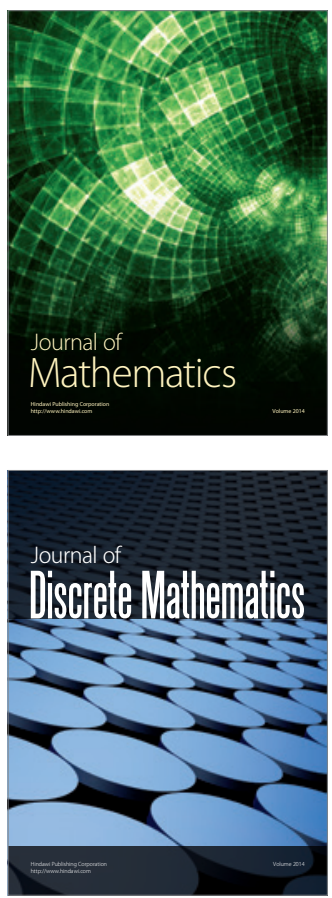

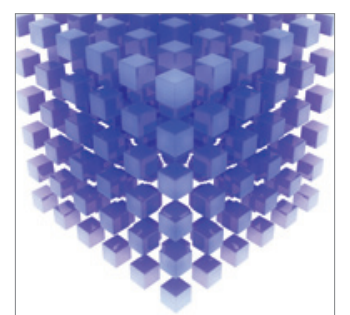

Mathematical Problems in Engineering
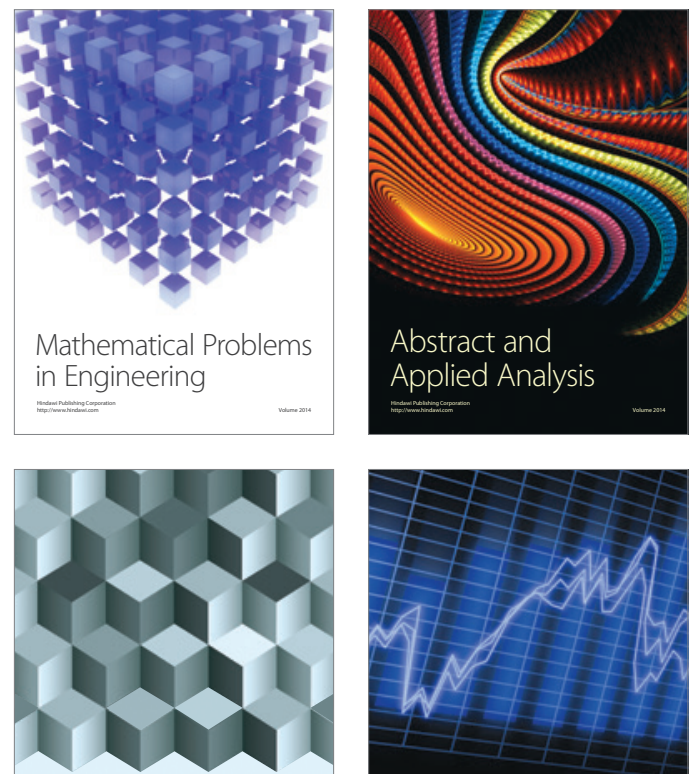

Journal of

Function Spaces

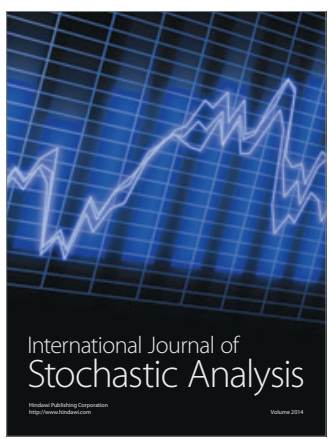

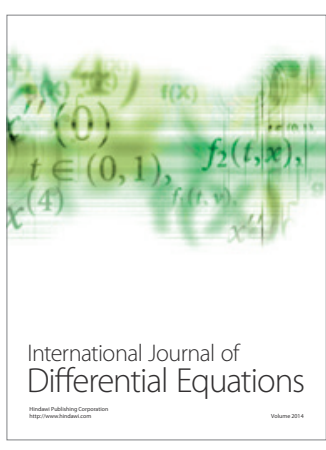
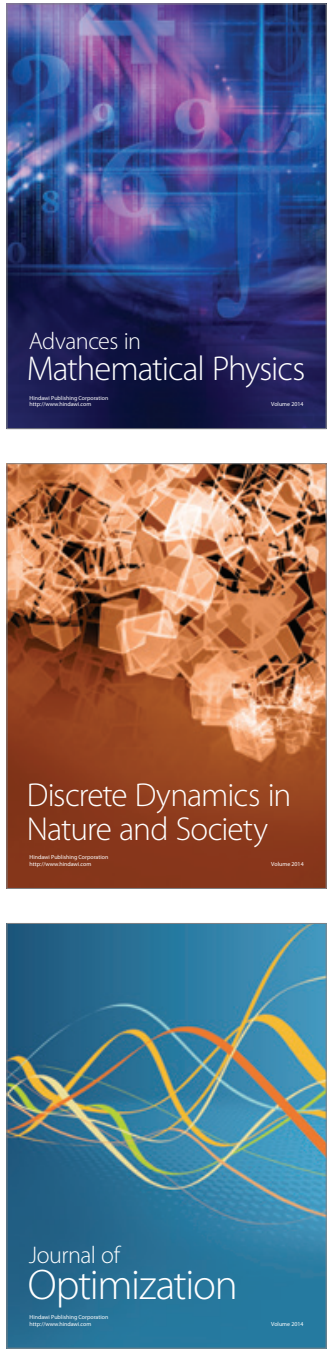